PROCEEDINGS OF THE

AMERICAN MATHEMATICAL SOCIETY

Volume 132, Number 10, Pages 3007-3018

S 0002-9939(04)07273-9

Article electronically published on June 2, 2004

\title{
ON THE EXISTENCE OF EIGENVALUES OF TOEPLITZ OPERATORS ON PLANAR REGIONS
}

\author{
CYRUS P. ARYANA AND KEVIN F. CLANCEY
}

(Communicated by Joseph A. Ball)

\begin{abstract}
A study is made of the eigenvalues of self-adjoint Toeplitz operators on multiply connected planar regions having $g$ holes. The presence of eigenvalues is detected through an analysis of the zeros of translations of theta functions restricted to $\mathbb{R}^{g}$ in $\mathbb{C}^{g}$.
\end{abstract}

\section{INTRODUCTION}

The theory of Toeplitz operators on multiply connected planar regions $D$ having $g$ holes acting on the Hardy spaces $H^{2}\left(d m_{a}\right)$ with respect to harmonic measures $m_{a}$ (based at a fixed point $a$ in $D$ ) has been investigated by M. B. Abrahamse [1]. Among his results is the following example: let $D$ be the annulus $D_{r}=\{z: r<|z|<$ $1\}$ with the outer boundary $b_{0}$, inner boundary $b_{1}$ and let $\phi \equiv 1$ on $b_{0}, \phi \equiv-1$ on $b_{1}$. Let $T_{\phi}$ be the corresponding self-adjoint Toeplitz operator on $H^{2}\left(d m_{a}\right)$. Then the essential spectrum of $T_{\phi}$ is given by $\sigma_{e}\left(T_{\phi}\right)=\{-1,1\}$. In fact, Abrahamse shows that $-1,1$ cannot be eigenvalues of $T_{\phi}$ and, hence, there exist two sequences $\left\{\lambda_{n}^{ \pm}\right\}$of eigenvalues in the "gap" $(-1,1)$ in the range of $\phi$ such that $\lambda_{n}^{ \pm} \rightarrow \pm 1$ and $\sigma\left(T_{\phi}\right)=\{-1,1\} \cup\left\{\lambda_{n}^{ \pm}: n=1,2, \ldots\right\}$. The explicit determination of $\lambda_{n}^{ \pm}$is in Clancey [3] $, \lambda_{n}^{ \pm}=\frac{q_{0}+p_{1} r^{2 n}}{q_{0}-p_{1} r^{2 n}}, n \in \mathbb{Z}$, where $q_{0}$ is the pole of the Green's function for $D_{r}$ in the interval $(r, 1)$ and $p_{1}$ is the critical value of the Green's function for $D_{r}$ with pole at $q_{0}$. Self-adjoint Toeplitz operators on multiply connected regions have also been studied by Pincus and Xia [8]. Among other things they give the estimate $\operatorname{dim} \operatorname{Ker}\left(T_{\phi}-\lambda\right) \leq g$, where $g$ is the number of holes.

It is also known (see, e.g., [3]), when the symbol $\phi$ (not necessarily real) is Hölder continuous on $\partial D$, that the multiplicity of the zero of an appropriate theta function determines the dimension of $\operatorname{Ker}\left(T_{\phi}-\lambda\right)$.

One interesting problem is to determine a necessary and sufficient condition for the existence of infinitely many eigenvalues of self-adjoint Toeplitz operators $T_{\phi}$ in the gaps in the range of the symbol $\phi$. This paper is focused on this problem in the cases when $g=1,2$. The analysis here uses a resolvent formula for self-adjoint Toeplitz operators on multiply connected planar regions. This resolvent formula is expressed in terms of theta functions associated with double $[4 ; 2]$. Consequently, our investigation leads to the study of zeros of the theta functions. Moreover, when

Received by the editors June 5, 2002 and, in revised form, March 7, 2003.

2000 Mathematics Subject Classification. Primary 47B35.

Key words and phrases. Double, harmonic measure, theta function, Hardy space, Toeplitz operator. 
the genus $g=2$, we need to give an explicit description of the loci of the zeros of the theta functions on real horizontal planes through half periods. The case when $g=1$ is resolved completely and a sufficient condition for the case $g=2$ is provided.

This paper is organized as follows. In Section 1 we will review some preliminaries concerning doubles of multiply connected planar regions as compact Riemann surfaces and their associated theta functions. Moreover, some introductory discussions about Toeplitz operators on the least harmonic majorant Hardy spaces and their resolvent formulae will be made in this section. In Theorem 1 of Section 2 we will present a necessary and sufficient condition for the existence of infinitely many eigenvalues in the gaps in the range of the symbol $\phi$. The proof of Theorem 1 will be given in this section. In Section 3 we will give an explicit description of the zeros of the theta functions on real horizontal planes through half periods. In Section 4 , when $g=2$, we will establish a sufficient condition for the existence of infinite sequences of eigenvalues in the gaps.

\section{Preliminaries}

Let $D$ be a bounded multiply connected planar region having $g \geq 1$ holes with analytic boundaries $b_{1}, \ldots, b_{g}$, and let $b_{0}$ be the boundary of the unbounded component of the complement of $D$. Let $X$ be the double of $D$ marked by the symmetric canonical homology basis $a_{1}, \ldots, a_{g}: b_{1}, \ldots, b_{g}$, where $a_{i}=\alpha_{i}-J \alpha_{i}$, and $\alpha_{i}$ is a cross cut from a fixed point $p_{0}$ on $b_{0}$ to a point on $b_{i}, i=1, \ldots, g$. Let $\left\{d w_{1}, \ldots, d w_{g}\right\}$ be the basis for the space $\Omega(X)$ of holomorphic differentials dual $\left(\int_{\alpha_{i}} d w_{j}=\delta_{i j}\right)$ to the above canonical homology basis with the "B-period" matrix $\tau=i P$. Let $m_{a}$ be the harmonic measure based at a fixed point $a$ in $D$. The least harmonic majorant Hardy space $H^{2}\left(d m_{a}\right)$ associated with $m_{a}$ is the closure in $L^{2}\left(d m_{a}\right)$ of the functions holomorphic on the closure of $D$. The Hardy spaces $H^{2}\left(d m_{a}\right)$ are reproducing kernel Hilbert spaces. This means that for a given $z$ in $D$, there is an element $k_{z}$ in $H^{2}\left(d m_{a}\right)$ satisfying $f(z)=\left\langle f, k_{z}\right\rangle$, for any $f$ in $H^{2}\left(d m_{a}\right)$, where $\langle\cdot, \cdot\rangle$ denotes the inner product in $H^{2}\left(d m_{a}\right)$.

The theta function associated with $\tau$ is the entire function on $\mathbb{C}^{g}$ defined by

$$
\theta(\underline{z})=\theta(\underline{z}, \tau)=\sum_{\underline{n} \in \mathbb{Z}^{g}} \exp \left\{2 \pi i\left(\frac{1}{2} \underline{n}^{t} \tau \underline{n}+\underline{n}^{t} \underline{z}\right)\right\} .
$$

Here we consider elements $\underline{z} \in \mathbb{C}^{g}$ and $\underline{n} \in \mathbb{Z}^{g}$ as column vectors and " $t$ " denotes transpose. This function is quasi-periodic in the sense that for $\underline{z} \in \mathbb{C}^{g}$ and $\underline{m}, \underline{n}$ in $\mathbb{Z}^{g}$

$$
\theta\left(\underline{z}+\underline{m}+\underline{n}^{t} \tau\right)=\exp \left\{2 \pi i\left(-\frac{1}{2} \underline{n}^{t} \tau \underline{n}-\underline{n}^{t} \underline{z}\right)\right\} \theta(\underline{z}) .
$$

In particular, $\theta$ is $\mathbb{Z}^{g}$ periodic. The quasi-periodicity of the theta function $\theta$ implies the invariance of the zero set $\theta_{0}$ of $\theta$ under translation by elements in the period lattice $\mathbb{Z}^{g}+\tau \mathbb{Z}^{g}$. Therefore, $\left[\theta_{0}\right]=\{[\underline{z}]: \theta(\underline{z})=0\}$ is a well-defined subvariety of the complex torus $\operatorname{Jac}(X)=\mathbb{C}^{g} /\left(\mathbb{Z}^{g}+\tau \mathbb{Z}^{g}\right)$.

Recall that the Abel-Jacobi map $\Phi_{0}$ based at a fixed point $p_{0}$ on $b_{0}$ is a map from $X$ to the Jacobian variety $\operatorname{Jac}(X)$ defined by

$$
\Phi_{0}(p)=\int_{p_{0}}^{p} d \vec{w} \bmod \left(\mathbb{Z}^{g}+\tau \mathbb{Z}^{g}\right),
$$


where $d \vec{w}=\left(d w_{1}, \ldots, d w_{g}\right)^{t}$. Since the B-period matrix of the marked double $X$ has the form $\tau=i P$, where $P$ is a real $g \times g$ positive definite symmetric matrix, the antiholomorphic involution map $J$ defined on the Jacobian variety by $J([\underline{z}])=-[\underline{z}]$ is a well-defined map.

Let $W_{d}$ denote the image in $\operatorname{Jac}(X)$ under $\Phi_{0}$ of the collection of non-negative divisors of degree $d(\geq 1)$, and let $W_{0}=\{0\}$. The following theorem of Riemann 6] characterizes the zero locus of the theta function associated with $\tau$ : When the genus of the marked double $X$ is $g \geq 1,\left[\theta_{0}\right]$ satisfies $\left[\theta_{0}\right]=W_{g-1}+\left[\Delta_{0}\right]$, where $\Delta_{0}$ is a constant called the Riemann constant. The explicit form of $\left[\Delta_{0}\right]$ is provided below.

Let $P$ denote the orthogonal projection of $L^{2}\left(d m_{a}\right)$ onto $H^{2}\left(d m_{a}\right)$. Given $\phi$ in $L^{\infty}\left(d m_{a}\right)$ one defines the Toeplitz operator $T_{\phi}$ with the symbol $\phi$ on $H^{2}\left(d m_{a}\right)$ by

$$
T_{\phi} f=P(\phi f), \quad f \in H^{2}\left(d m_{a}\right) .
$$

The Toeplitz operator $T_{\phi}$ is self-adjoint if and only if the symbol $\phi$ is real-valued. The resolvent formula for the self-adjoint Toeplitz operators is given in [4] as follows. Let $\phi$ be a real-valued element in $L^{\infty}\left(d m_{a}\right)$, and let $z$ be given in $D$. Then for the reproducing kernel $k_{z}$ in $H^{2}\left(d m_{a}\right)$ and for any complex number $\lambda$ satisfying $\operatorname{Im} \lambda \neq 0$, there holds

$$
\begin{aligned}
& \left\langle\left(T_{\phi}-\lambda\right)^{-1} k_{z}, k_{z}\right\rangle \\
& =C(z) \frac{\theta\left(\omega_{z}+t_{a}-\frac{1}{2 \pi i} \int_{\partial D} \log (\phi-\lambda) d \vec{w}\right)}{\theta\left(t_{a}-\frac{1}{2 \pi i} \int_{\partial D} \log (\phi-\lambda) d \vec{w}\right)} e^{-\int_{\partial D} \log (\phi-\lambda) d m_{z}},
\end{aligned}
$$

where $t_{a}$ is a constant real number and $C(z), \omega_{z}$ are constants depending only on $z$.

We need here to mention the fact that $d \vec{w}=\frac{1}{2} d \vec{\omega}+\frac{i}{2} * d \vec{\omega}=\frac{i}{2} * d \vec{\omega}$, on $\partial D$, where $\omega_{j}=\omega_{j}(z)(0 \leq j \leq g)$ is the harmonic measure of $b_{j}$ based at $z$. We will denote the signed measure $\frac{-1}{2} * d \omega_{j}$ by $d \nu_{j}$. In fact, the holomorphic differential $d w_{j}$ is the reflection of the holomorphic differential $\partial \omega_{j} d z$ from $\bar{D}$ on the double $X$.

We close this section with the remark that the theta function associated with a double of genus $g$ never vanishes in $\mathbb{R}^{g}$. This is a result of Fay [7, p. 118]. Moreover, in the case $g=1$ the associated theta function vanishes only at the point $\frac{1}{2}+\frac{1}{2} \tau$ and its translations by $\mathbb{Z}+\tau \mathbb{Z}$.

It will be convenient to use the following terminology. Given $\phi$ in $L^{\infty}\left(d m_{a}\right)$ the open intervals forming the bounded components of the complement of the essential range of $\phi$ will be called gaps.

\section{TheOREM 1}

From the functional calculus for a self-adjoint operator it follows that the isolated singularities of the resolvent are eigenvalues. Moreover, if $T$ is a self-adjoint operator with eigenvector $f$ corresponding to the eigenvalue $z=a$, then whenever $\langle f, k\rangle$ is non-zero, the restriction of $T$ to the smallest invariant subspace containing $k$ will have eigenvalue $z=a$. Since $k_{z}, z \in D$ is dense in $H^{2}\left(d m_{a}\right)$, one can see from the resolvent formula that the zeros of the function

$$
f(t)=\theta\left(t_{a}-\frac{1}{2 \pi i} \int_{\partial D} \log (\phi-t) d \vec{w}\right)
$$


in a gap are precisely the eigenvalues of $T_{\phi}$ in the gap. We can use the work of Riemann to describe in $\mathbb{C}^{g}$ the zero subvariety for the theta function $\theta$ associated with the marked double $X$ of genus $g \geq 1$. Moreover, since the Hardy spaces are conformally invariant, to study the existence of eigenvalues of the self-adjoint Toeplitz operators $T_{\phi}$ on the Hardy spaces $H^{2}\left(d m_{a}\right)$ of the $g$-holed $D$, one can replace the region $D$ by its conformal model, which can be chosen as a circular region obtained from the unit disc by removing $g$ disjoint closed discs. When $g=1$, one can choose the region $D$ to be an annulus.

Theorem 1. Suppose that $D$ is the annulus $D_{r}=\{z: r<|z|<1\}$, and suppose that $\phi \in L^{\infty}\left(d m_{a}\right), \phi=\bar{\phi}$. Let $m_{i}=\operatorname{essinf}_{t \in b_{i}} \phi(t), M_{i}=\operatorname{esssup}_{t \in b_{i}} \phi(t), i=$ 0,1 . Assume that the intervals $\left[m_{0}, M_{0}\right]$ and $\left[m_{1}, M_{1}\right]$ are disjoint. Without loss of generality, we assume that $M_{1}<m_{0}$. Then there exist sequences $\left\{\lambda_{n}\right\}_{n=1}^{\infty}$ of eigenvalues in the gap $\left(M_{1}, m_{0}\right)$ converging to $M_{1}$ (respectively, $\left.m_{0}\right)$ if and only if

$$
\int_{0}^{2 \pi} \log \left|\phi\left(r e^{i \theta}\right)-M_{1}\right| \frac{d \theta}{2 \pi}=-\infty\left(\text { respectively, } \int_{0}^{2 \pi} \log \left|\phi\left(e^{i \theta}\right)-m_{0}\right| \frac{d \theta}{2 \pi}=-\infty\right) .
$$

Proof. When $t$ is in the gap $\left(M_{1}, m_{0}\right)$ of the spectrum of $T_{\phi}$, the function $f(t)=$ $\theta\left(t_{a}-\frac{1}{2 \pi i} \int_{\partial D} \log (\phi-t) d w\right)$ associated with the function in the denominator of the resolvent formula is in the form $f(t)=\theta\left(x(t)+\frac{1}{2} \tau\right)$, where $x(t)=t_{a}+$ $\frac{1}{2 \pi} \int_{\partial D} \log |\phi-t| d \nu, t \in$ (gap). Therefore, one needs to study the intersections of zero locus of the theta function $\theta$ in the real hyperplane $P=\left\{z=x+\frac{1}{2} \tau: x \in \mathbb{R}\right\}$ with the path $x(t)+\frac{1}{2} \tau$. The zero locus of $\theta$ in $P$ is the set of $m+\frac{1}{2}+\frac{1}{2} \tau$, $m \in \mathbb{Z}$. Thus, the proof reduces to the study of a necessary and sufficient condition for the validity of the equation $x(t)=m+\frac{1}{2}$ infinitely often. One can see that $\int_{b_{1}} \log \left|\phi-M_{1}\right| d \nu=-\infty$ if and only if $\int_{0}^{2 \pi} \log \left|\phi\left(r e^{i \theta}\right)-M_{1}\right| \frac{d \theta}{2 \pi}=-\infty$. Continuity of the path $x(t)$ implies that as $t$ approaches $M_{1}$ from the right, the equation $x(t)=m+\frac{1}{2}$ will hold infinitely often if and only if $\int_{0}^{2 \pi} \log \left|\phi\left(r e^{i \theta}\right)-M_{1}\right| \frac{d \theta}{2 \pi}=-\infty$. Similarly, as $t$ approaches $m_{0}$ from the left, the path $x(t)$ will hit the zero locus of $\theta$ infinitely often. The proof of the theorem is complete.

\section{Proposition 1}

If the region $D$ is obtained from the unit disc by removing $g$ disjoint closed discs centered on the real axis, the double $X$ of such a region has the extra anti-conformal map $Q: X \rightarrow X$ defined by reflection in the real axis. In such a case the Riemann constant $\Delta_{0}$ for $X$ based at $p_{0}=-1$ has the explicit form

$$
\Delta_{0}=\frac{1}{2}\left[\begin{array}{c}
1 \\
1 \\
\vdots \\
1
\end{array}\right]+\frac{1}{2} \tau\left[\begin{array}{c}
g \\
g-1 \\
\vdots \\
1
\end{array}\right]
$$

See [5. p. 310], or [2, p. 31] for a detailed computation. When $g$ is 2 it is always possible to conformally map $D$ to such a "conjugate symmetric" circular region. In particular, the Riemann constant $\Delta_{0}$ for the double $X$ of genus two can be assumed to have the form

$$
\Delta_{0}=\frac{1}{2}\left[\begin{array}{l}
1 \\
1
\end{array}\right]+\frac{1}{2} \tau\left[\begin{array}{l}
0 \\
1
\end{array}\right]
$$




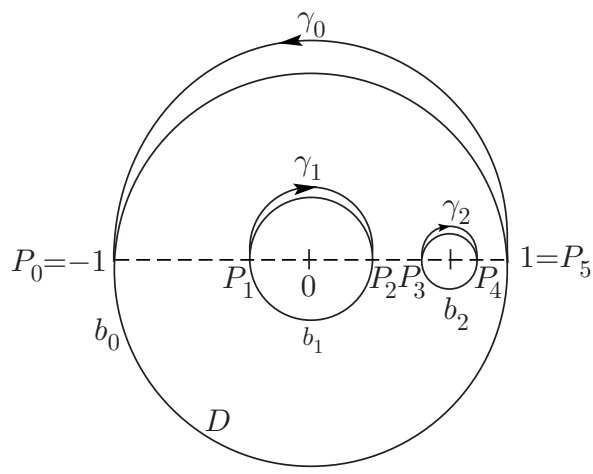

(a)

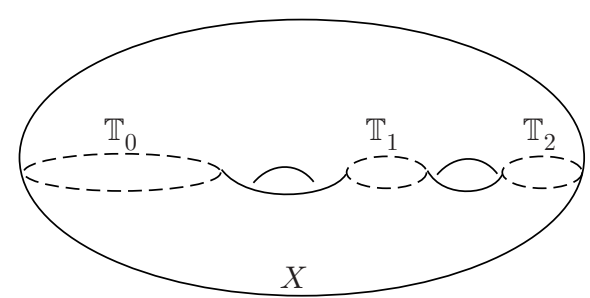

(b)

Figure 1.

The result we will describe here is for genus $g=2$. We choose $D$ to be as in Figure 1(a). The notation $\mathbb{T}_{0}, \mathbb{T}_{1}$ and $\mathbb{T}_{2}$ will be used for the "circles" in $X$ formed by doubling the intervals $I_{0}=\left(p_{0}, p_{1}\right), I_{1}=\left(p_{2}, p_{3}\right)$ and $I_{2}=\left(p_{4}, p_{5}\right)$, respectively. See Figure 1(b).

We will see that we need only to look for zeros of theta functions in the real horizontal planes $P_{\underline{\lambda}}=\left\{\underline{z}=\underline{x}+\frac{1}{2} \tau \underline{\lambda}: \underline{x} \in \mathbb{R}^{2}\right\}$ through the half periods $\frac{1}{2} \tau \underline{\lambda}$, $\underline{\lambda} \in \mathbb{Z}^{2} / 2 \mathbb{Z}^{2}$. Since $\underline{\lambda}=\left[\begin{array}{l}\lambda_{1} \\ \lambda_{2}\end{array}\right] \in \mathbb{Z}^{2} / 2 \mathbb{Z}^{2}$, there are $2^{2}$ of these planes. Moreover, $\theta$ maps $\mathbb{R}^{2}$ into $\mathbb{R}^{*}$. Therefore, the zero locus corresponding to $\underline{\lambda}=\left[\begin{array}{l}0 \\ 0\end{array}\right]$ is the empty set. Thus, it remains to study 3 of these planes. Since theta functions are quasi-periodic, this leads to the study of the zeros of only the following functions in $\mathbb{R}^{2}$ :

$$
\begin{array}{ll}
\text { (I) } \quad F(\underline{x})=\theta\left(\underline{x}+\frac{1}{2} \tau\left[\begin{array}{l}
0 \\
1
\end{array}\right]\right), \quad \underline{x} \in \mathbb{R}^{2} ; \\
(\mathrm{II}) \quad G(\underline{x})=\theta\left(\underline{x}+\frac{1}{2} \tau\left[\begin{array}{l}
1 \\
0
\end{array}\right]\right), \quad \underline{x} \in \mathbb{R}^{2} ; \\
(\mathrm{III}) \quad H(\underline{x})=\theta\left(\underline{x}+\frac{1}{2} \tau\left[\begin{array}{l}
1 \\
1
\end{array}\right]\right), \quad \underline{x} \in \mathbb{R}^{2} .
\end{array}
$$

The discussion below will show that the zero loci of the above functions $F, G$, and $H$ are as in the following Figures 2(a), 2(b), and 2(c), respectively.

One determines the zero set of $F$ using the following:

Proposition 1. The complete zero set of the function F given by (I) is the union of the arcs with the following parameterization:

$$
\left[\begin{array}{c}
\frac{1}{2} \omega_{1}(p)+k+\frac{1}{2} \\
\frac{1}{2} \omega_{2}(p)+\frac{1}{2}
\end{array}\right], k \in \mathbb{Z}
$$

where $p$ varies over $\mathbb{T}_{0}$. 


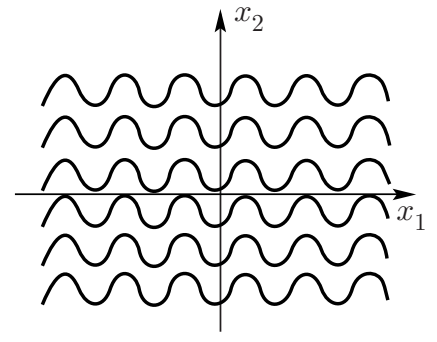

(a)

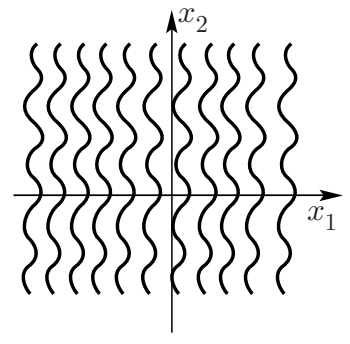

(b)

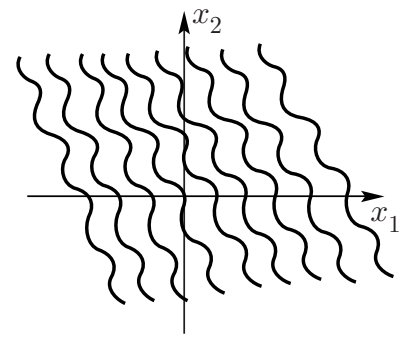

(c)

FIGURE 2 .

Proof. Referring to $(*), F(\underline{x})=\theta\left(\underline{x}+\frac{1}{2} \tau\left[\begin{array}{l}0 \\ 1\end{array}\right]\right)=0 \Leftrightarrow \theta\left(\underline{x}-\frac{1}{2}\left[\begin{array}{l}1 \\ 1\end{array}\right]+\Delta_{0}\right)=0$. By Riemann's characterization of zero set of theta (Theorem VI.3.1 in [6]), this last condition is equivalent to $\underline{x}-\frac{1}{2}\left[\begin{array}{l}1 \\ 1\end{array}\right]+\Delta_{0}=\int_{p_{0}}^{p} d \vec{w} \bmod \left(\mathbb{Z}^{2}+\tau \mathbb{Z}^{2}\right)+\Delta_{0}$, for some $p$. Cancelling out $\Delta_{0}$ from both sides, the last condition is equivalent to $\int_{p_{0}}^{p} d \vec{w} \in \mathbb{R}^{2} /\left(\mathbb{Z}^{2}+\tau \mathbb{Z}^{2}\right)$, for some path from $p_{0}$ to $p$. Using the fact that $d \vec{w}=\frac{1}{2}(d \vec{\omega}+i * d \vec{\omega})$, we see that this last condition is equivalent to $\left[\int_{p_{0}}^{p} * d \vec{\omega}\right]=$ $[\underline{0}]$, for some path from $p_{0}$ to $p$. If it is so, then $\underline{x}=\operatorname{Re}\left(\int_{p_{0}}^{p} d \vec{w}+\frac{1}{2}\left[\begin{array}{l}1 \\ 1\end{array}\right]\right)\left(\bmod \mathbb{Z}^{2}\right)$. If the path of integration is taken along the loop $\mathbb{T}_{0}$, Figure $1(\mathrm{~b})$, then $\int_{p_{0}}^{p} d \vec{w}$ is a real vector (and hence $\int_{p_{0}}^{p} * d \vec{\omega}=\underline{0}$ ). When $p$ traces the loop $\mathbb{T}_{0}$ for the first time,

$$
\int_{p_{0}}^{p} d \vec{w}=\frac{1}{2}\left[\begin{array}{l}
\omega_{1}(p)-\omega_{1}\left(p_{0}\right) \\
\omega_{2}(p)-\omega_{2}\left(p_{0}\right)
\end{array}\right]=\frac{1}{2}\left[\begin{array}{l}
\omega_{1}(p) \\
\omega_{2}(p)
\end{array}\right] \in \mathbb{R}^{2},
$$

and when $p$ traces $\mathbb{T}_{0}$ for the $k$ th time, $k \in \mathbb{Z}^{*}$ (here, $k<0$ denotes $-k$ revolutions in the negative direction),

$$
\int_{p_{0}}^{p} d \vec{w}=\frac{1}{2}\left[\begin{array}{l}
\omega_{1}(p) \\
\omega_{2}(p)
\end{array}\right]+\left[\begin{array}{c}
k \\
0
\end{array}\right]=\left[\begin{array}{c}
\frac{1}{2} \omega_{1}(p)+k \\
\frac{1}{2} \omega_{2}(p)
\end{array}\right] .
$$

Since $\vec{\omega}(p)$ is a continuous map, the image of $p$ under the map $\int_{p_{0}}^{p} d \vec{w}$ is a curve with the parameterization $\left[\begin{array}{c}\frac{1}{2} \omega_{1}(p)+k \\ \frac{1}{2} \omega_{2}(p)\end{array}\right], k \in \mathbb{Z}^{*}$. Therefore, the translation of the image of $\int_{p_{0}}^{p} d \vec{w}$ by $\frac{1}{2}\left[\begin{array}{l}1 \\ 1\end{array}\right]$ has the parameterization

$$
\left[\begin{array}{c}
\frac{1}{2} \omega_{1}(p)+k+\frac{1}{2} \\
\frac{1}{2} \omega_{2}(p)+\frac{1}{2}
\end{array}\right], k \in \mathbb{Z}
$$

Note that the second component, $\frac{1}{2} \omega_{2}(p)+\frac{1}{2}$ has the same behavior in each revolution. Furthermore, $0 \leq \frac{1}{2} \omega_{2}(p)+\frac{1}{2} \leq 1$ for all $p$. Thus, this curve lies in a horizontal strip of one unit width. By plotting the points $\left[\begin{array}{l}\frac{1}{2} \omega_{1}\left(p_{0}\right)+\frac{1}{2} \\ \frac{1}{2} \omega_{2}\left(p_{0}\right)+\frac{1}{2}\end{array}\right]=\left[\begin{array}{c}\frac{1}{2} \\ \frac{1}{2}\end{array}\right]$, 
$\left[\begin{array}{c}\frac{1}{2} \omega_{1}\left(p_{1}\right)+\frac{1}{2} \\ \frac{1}{2} \omega_{2}\left(p_{1}\right)+\frac{1}{2}\end{array}\right]=\left[\begin{array}{c}1 \\ \frac{1}{2}\end{array}\right]$, and $\left[\begin{array}{c}\frac{1}{2} \omega_{1}\left(p_{0}\right)+1+\frac{1}{2} \\ \frac{1}{2} \omega_{2}\left(p_{0}\right)+\frac{1}{2}\end{array}\right]=\left[\begin{array}{l}3 / 2 \\ 1 / 2\end{array}\right]$, using the above parameterization and $\mathbb{Z}^{2}$ periodicity of $F$, we get a portion of the zero divisor of $F$ in $\mathbb{R}^{2}$ as in Figure 2(a). We claim that this locus is the complete locus of all zeros of $F$. To establish this, it is sufficient to show that $\int_{p_{0}}^{p} * d \vec{\omega} \neq \underline{0}$, whenever $p$ is off the loop $\mathbb{T}_{0}$. The function $\tilde{\omega}_{1}(p)=\int_{p_{0}}^{p} * d \omega_{1}$ is a single-valued continuous function on $\bar{D}$ and harmonic in $\mathcal{G}=D \backslash\left\{I_{1} \cup I_{2}\right\}$ if the path of integration does not go around any boundary components. Moreover, it is zero on $I_{0}$. For the remainder of the proof we use the notation $\gamma_{0}, \gamma_{1}, \gamma_{2}$ as in Figure 1(a). Under the above condition on the path of integration, since the restriction to the boundary $\partial D$ of the differential $* d \omega_{1}=\frac{\partial \omega_{1}}{\partial \eta} d s$, and $\frac{\partial \omega_{1}}{\partial \eta}>0$ on $\gamma_{1}, \frac{\partial \omega_{1}}{\partial \eta}<0$ on $\gamma_{0}, \gamma_{2}$, one can see that $\tilde{\omega}_{1}(p) \geq 0$ on $I_{0} \cup \gamma_{1} \cup I_{1} \cup \gamma_{2} \cup I_{2} \cup \gamma_{0}$. Note that the orientation of the boundary is consistent with the orientation for the arc length. Thus,

$$
\int_{p_{0}}^{p_{5}} * d \omega_{1}=-\int_{\gamma_{0}} * d \omega_{1}=-\int_{\gamma_{0}} \frac{\partial \omega_{1}}{\partial \eta} d s>0 .
$$

Therefore, using the maximum principle, $\tilde{\omega}_{1}$ never vanishes on $\mathcal{G}$ intersected with the upper half-plane. Using the symmetry of the region and the anti-conformal involution map $J$, one can show that $\tilde{\omega}_{1}$ will never vanish off the loop $\mathbb{T}_{0}$. This proves that the above zero locus is the complete zero divisor of $F(\underline{x})$.

In a similar manner one sees that the complete zero sets of $G$ and $H$ are the union of the arcs with parameterizations

$$
\left[\begin{array}{c}
\frac{1}{2} \omega_{1}(p)+\frac{1}{2} \\
\frac{1}{2} \omega_{2}(p)+\frac{1}{2}+k
\end{array}\right], k \in \mathbb{Z}, \quad \text { and } \quad\left[\begin{array}{c}
\frac{1}{2} \omega_{1}(p)-k+\frac{1}{2} \\
\frac{1}{2} \omega_{2}(p)+k+\frac{1}{2}
\end{array}\right], k \in \mathbb{Z}
$$

respectively, where $p$ varies over $\mathbb{T}_{2}$ and $\mathbb{T}_{1}$, respectively. The details of these cases are given in [2].

Remark. As can be seen from the Figures 2(a), 2(b), 2(c), the zero sets of $F, G, H$ have the following geometric property. A line in $\mathbb{R}^{2}$ with positive slope will intersect these zero sets infinitely often.

\section{Theorem 2}

Let the genus $g=2$. In the sequel we assume that $\phi \in L^{\infty}\left(d m_{a}\right), \phi=\bar{\phi}$, and we put $m_{i}=\operatorname{essinf}_{t \in b_{i}} \phi(t), M_{i}=\operatorname{esssup}_{t \in b_{i}} \phi(t), i=0,1,2$. Also, we assume that the intervals $\left[m_{0}, M_{0}\right],\left[m_{1}, M_{1}\right]$, and $\left[m_{2}, M_{2}\right]$ are separated by gaps. This means they are mutually disjoint. We are going to establish a sufficient condition for the existence of sequences of eigenvalues of $T_{\phi}$ in the gaps in the range of $\phi$.

When $t$ is in one of the gaps, the function $f(t)$, in Section 2, is in the form $f(t)=\theta\left(\underline{x}(t)+\frac{1}{2} \tau \underline{\lambda}\right)$, where $\underline{\lambda} \in \mathbb{Z}^{2} / 2 \mathbb{Z}^{2}$, and $\underline{x}(t)=t_{a}+\frac{1}{2 \pi} \int_{\partial D} \log |\phi-t| d \vec{\nu}$, $t \in$ (gap). Therefore, using the theorem of Riemann, one needs to investigate the intersections of zero loci of the theta function $\theta$ in the real horizontal planes $P_{\underline{\lambda}}=\left\{\underline{z}=\underline{x}+\frac{1}{2} \tau \underline{\lambda}: \underline{x} \in \mathbb{R}^{2}\right\}$ with the path $\underline{x}(t)=t_{a}+\frac{1}{2 \pi} \int_{\partial D} \log |\phi-t| d \vec{\nu}, t \in$ (gap).

Using the notation introduced in the preceding section, one needs only to study the intersections of the zero loci of the theta functions $F, G$, and $H$, from Section 3 , in $\mathbb{R}^{2}$ with the path $\underline{x}(t)$. 
TABLE 1.

\begin{tabular}{|c|c|c|c|}
\hline order of intervals & gap & $\theta\left(t_{a}-\frac{1}{2 \pi} \int_{\partial D} \log (\phi-t) d \vec{w}\right)$ & existence of eigenvalues \\
\hline$M_{2}<m_{1} \leq M_{1}<m_{0}$ & $M_{2}<m_{1}$ & $\theta\left(\underline{x}(t)-\frac{1}{2} \tau\left[\begin{array}{l}0 \\
1\end{array}\right]\right)$ & two infinite sequences \\
\cline { 2 - 4 } & $M_{1}<m_{0}$ & $\theta\left(\underline{x}(t)-\frac{1}{2} \tau\left[\begin{array}{l}1 \\
1\end{array}\right]\right)$ & at least one infinite sequence \\
\hline \multirow{3}{*}{$M_{1}<m_{2} \leq M_{2}<m_{0}$} & $M_{1}<m_{2}$ & $\theta\left(\underline{x}(t)-\frac{1}{2} \tau\left[\begin{array}{l}1 \\
0\end{array}\right]\right)$ & two infinite sequences \\
\cline { 2 - 4 } & $M_{2}<m_{0}$ & $\theta\left(\underline{x}(t)-\frac{1}{2} \tau\left[\begin{array}{l}1 \\
1\end{array}\right]\right)$ & at least one infinite sequence \\
\hline \multirow{3}{*}{$M_{2}<m_{0} \leq M_{0}<m_{1}$} & $M_{2}<m_{0}$ & $\theta\left(\underline{x}(t)-\frac{1}{2} \tau\left[\begin{array}{c}0 \\
1\end{array}\right]\right)$ & two infinite sequences \\
\cline { 2 - 4 } & $M_{0}<m_{1}$ & $\theta\left(\underline{x}(t)-\frac{1}{2} \tau\left[\begin{array}{c}-1 \\
0\end{array}\right]\right)$ & two infinite sequences \\
\hline \multirow{3}{*}{$M_{1}<m_{0} \leq M_{0}<m_{2}$} & $M_{1}<m_{0}$ & $\theta\left(\underline{x}(t)-\frac{1}{2} \tau\left[\begin{array}{c}1 \\
0\end{array}\right]\right)$ & two infinite sequences \\
\cline { 2 - 5 } & $M_{0}<m_{2}$ & $\theta\left(\underline{x}(t)-\frac{1}{2} \tau\left[\begin{array}{c}0 \\
-1\end{array}\right]\right)$ & two infinite sequences \\
\hline \multirow{2}{*}{$M_{0}<m_{2} \leq M_{2}<m_{1}$} & $M_{0}<m_{2}$ & $\theta\left(\underline{x}(t)-\frac{1}{2} \tau\left[\begin{array}{l}-1 \\
-1\end{array}\right]\right)$ & at least one infinite sequence \\
\cline { 2 - 5 } & $M_{2}<m_{1}$ & $\theta\left(\underline{x}(t)-\frac{1}{2} \tau\left[\begin{array}{c}-1 \\
0\end{array}\right]\right)$ & two infinite sequences \\
\hline \multirow{2}{*}{$M_{0}<m_{1} \leq M_{1}<m_{2}$} & $M_{0}<m_{1}$ & $\theta\left(\underline{x}(t)-\frac{1}{2} \tau\left[\begin{array}{l}-1 \\
-1\end{array}\right]\right)$ & at least one infinite sequence \\
\cline { 2 - 5 } & $M_{1}<m_{2}$ & $\theta\left(\underline{x}(t)-\frac{1}{2} \tau\left[\begin{array}{c}0 \\
-1\end{array}\right]\right)$ & two infinite sequences \\
\hline
\end{tabular}

The following theorem covers the case where $M_{2}<m_{1} \leq M_{1}<m_{0}$.

Theorem 2. Suppose $D$ is a conjugate symmetric region with two holes. Let $\phi \in$ $L^{\infty}\left(d m_{a}\right), \phi=\bar{\phi}$. If $M_{2}<m_{1} \leq M_{1}<m_{0}$, then

(i) There exist two infinite sequences $\left\{\lambda_{n}\right\}_{n=1}^{\infty}$ of eigenvalues in the gap $\left(M_{2}, m_{1}\right)$, one converging to $M_{2}$ if $\int_{b_{2}} \log \left|\phi-M_{2}\right| d m_{a}=-\infty$ and the other converging to $m_{1}$ if $\int_{b_{1}} \log \left|\phi-m_{1}\right| d m_{a}=-\infty$.

(ii) There is an infinite sequence $\left\{\lambda_{n}\right\}_{n=1}^{\infty}$ of eigenvalues in the gap $\left(M_{1}, m_{0}\right)$, converging to $m_{0}$ if $\int_{b_{0}} \log \left|\phi-m_{0}\right| d m_{a}=-\infty$.

Below we will prove Theorem 2. For the convenience of the reader we collect the analogues of this theorem in Table 1. This table demonstrates all possible forms of the function $f(t)$, and the final column indicates the existence of an infinite sequence of eigenvalues in gaps and under the hypotheses that $\int_{b_{i}} \log \left|\phi-m_{i}\right| d m_{a}$ is infinity or $\int_{b_{i}} \log \left|\phi-M_{i}\right| d m_{a}$ is infinity.

Proof. (i): When $t$ is in the gap $\left(M_{2}, m_{1}\right)$ of the spectrum of $T_{\phi}$, the function $f(t)=\theta\left(t_{a}-\frac{1}{2 \pi i} \int_{\partial D} \log (\phi-t) d \vec{w}\right)$ associated with the function in the denominator of the resolvent formula is in the form $F(\underline{x}(t))=\theta\left(\underline{x}(t)+\frac{1}{2} \tau\left[\begin{array}{l}0 \\ 1\end{array}\right]\right)$, where

$$
\underline{x}(t)=\left[\begin{array}{l}
x_{1}(t) \\
x_{2}(t)
\end{array}\right]=t_{a}+\frac{1}{2 \pi} \int_{\partial D} \log |\phi-t| d \vec{\nu} \in \mathbb{R}^{2} .
$$

In Section 3, Figure 2(a), we exhibited the zero locus of the function in $F$ in $\mathbb{R}^{2}$. In order to see the intersection of the zero locus of the function $F$ with the path $\underline{x}(t)$ 
we consider that if $\int_{b_{2}} \log \left|\phi-M_{2}\right| d m_{a}=-\infty$, then $\int_{b_{2}} \log \left|\phi-M_{2}\right| d \vec{\nu}=\left[\begin{array}{l}+\infty \\ -\infty\end{array}\right]$. In fact, for $j=1,2$, we can write, for example,

$$
\int_{b_{2}} \log \left|\phi-M_{2}\right| d \nu_{j}=\int_{b_{2}} \log \left|\phi-M_{2}\right|\left(2 \pi i \frac{\nu_{j}}{\Omega_{a-J a}}\right) d m_{a}
$$

where $\Omega_{a-J a}$ is the normalized differential of the third kind with poles at $a, J a$ on the double $X$. The function $2 \pi i \frac{\nu_{j}}{\Omega_{a-J a}}$ is a symmetric multiple-valued meromorphic function having no poles on $\partial D$. The symmetric meromorphic differential $\frac{-1}{2 \pi i} \Omega_{a-J a}$ is positive on $\partial D$. Moreover, $\nu_{j}$ is positive on $b_{j}$ and negative on $b_{i}, i \neq j$. Hence, it is easy to see that

$$
\int_{b_{2}} \log \left|\phi-M_{2}\right| d m_{a}=-\infty \Longleftrightarrow \int_{b_{2}} \log \left|\phi-M_{2}\right| d \vec{\nu}=\left[\begin{array}{l}
+\infty \\
-\infty
\end{array}\right] .
$$

Therefore, it is easy to see that if the above hypotheses hold, then $\int_{b_{2}} \log |\phi-t| d \vec{\nu} \rightarrow$

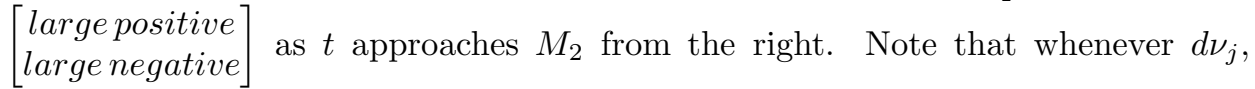
$j=1,2$, is considered only on one of the boundary components, it will be a positive or a negative measure. This property allows us to use the Lebesgue Dominated Convergence Theorem. Thus, if the above hypotheses hold, then the path $\underline{x}(t)=t_{a}-\frac{1}{2 \pi i} \int_{\partial D} \log |\phi-t| d \vec{w}$ intersects the above zero locus infinitely often as $t$ approaches $M_{2}$ from the right. Similarly, one can see that

$$
\int_{b_{1}} \log \left|\phi-m_{1}\right| d m_{a}=-\infty \Longleftrightarrow \int_{b_{1}} \log \left|\phi-M_{2}\right| d \vec{\nu}=\left[\begin{array}{l}
-\infty \\
+\infty
\end{array}\right] .
$$

Therefore, if the above hypotheses hold, then $\int_{b_{1}} \log |\phi-t| d \vec{\nu} \rightarrow\left[\begin{array}{l}\text { large negative } \\ \text { large positive }\end{array}\right]$ as $t$ approaches $m_{1}$ from the left. Thus, if $\int_{b_{1}} \log \left|\phi-m_{1}\right| d m_{a}=-\infty$, then $\int_{b_{1}} \log \left|\phi-m_{1}\right| d \vec{\nu}=\left[\begin{array}{c}-\infty \\ +\infty\end{array}\right]$ and hence the path $\underline{x}(t)=t_{a}-\frac{1}{2 \pi i} \int_{\partial D} \log |\phi-t| d \vec{w}$ intersects the above zero locus infinitely often as $t$ approaches $m_{1}$ from the left. See Figure 3.

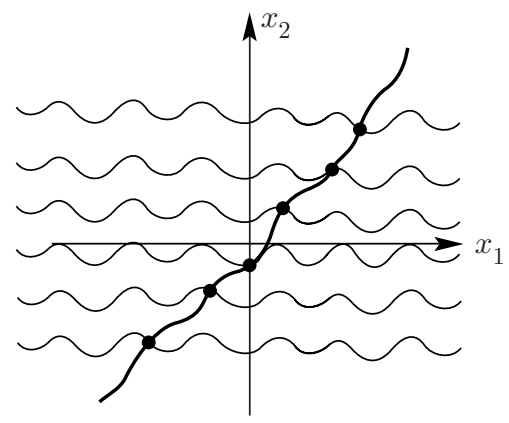

FIGURE 3 .

For each one of these zeros one can choose an appropriate $\omega_{z}$, by moving $z$ if necessary, so that the zero becomes an isolated singularity of the resolvent form. Note 


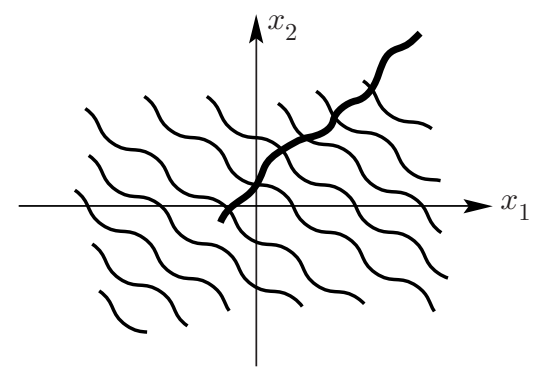

FIGURE 4.

that once we make a zero an isolated singularity this zero would be an eigenvalue of $T_{\phi}$.

(ii): When $t$ is in the gap $\left(M_{1}, m_{0}\right)$, the function

$$
f(t)=\theta\left(t_{a}-\frac{1}{2 \pi i} \int_{\partial D} \log (\phi-t) d \vec{w}\right)
$$

is in the form $f(t)=\theta\left(\underline{x}(t)+\frac{1}{2} \tau \underline{\lambda}\right)$, where $\underline{\lambda}=\left[\begin{array}{l}1 \\ 1\end{array}\right]$ and $\underline{x}(t)=\left[\begin{array}{l}x_{1}(t) \\ x_{2}(t)\end{array}\right] \in \mathbb{R}^{2}$. One can see that if $\int_{b_{0}} \log \left|\phi-m_{0}\right| d m_{a}=-\infty$, then $\int_{b_{0}} \log \left|\phi-m_{0}\right| d \vec{\nu}=\left[\begin{array}{c}+\infty \\ +\infty\end{array}\right]$. Thus,

$$
\int_{b_{0}} \log |\phi-t| d w_{j}=\int_{b_{0}} \log |\phi-t| d \nu_{j}, \quad j=1,2,
$$

gets large positive when $t$ approaches $m_{0}$ from the left. Therefore, referring to the picture of the zero locus of the theta function $G$ in $P_{\underline{\lambda}}$, Section 3, Figure 2(b), one can see that the path $\underline{x}(t)=t_{a}-\frac{1}{2 \pi i} \int_{\partial D} \log |\phi-t| d \vec{w}$ intersects the above zero locus infinitely often as $t$ approaches $m_{0}$ from the left, as in Figure 4 . This ends the proof of Theorem 2 .

Corollary 1. If $\phi$ is a simple function in $L^{\infty}\left(d m_{a}\right), \phi=\bar{\phi}$, and $M_{2}<m_{1} \leq$ $M_{1}<m_{0}$, then there always exist infinitely many eigenvalues of $T_{\phi}$ in the gaps as indicated in Table 1.

We close with an example that illustrates the effectiveness of the criteria that have been developed for detecting eigenvalues.

Example 1. Let $D$ be a region obtained from the open unit disc $\{z:|z|<1\}$ by removing 2 disjoint closed discs $\bar{D}_{1}, \bar{D}_{2}$ of radius $0<r_{0}<\frac{1}{2}$, centered at the points $-\frac{1}{2}$ and $\frac{1}{2}$, respectively, in the real axis, as in Figure 5 . Let $m_{a}$ be the harmonic measure based at a fixed point $a$ in $D$, and let $\phi$ be an element in $L^{\infty}\left(d m_{a}\right)$, $\bar{\phi}=\phi$, with $\phi_{i}=\left.\phi\right|_{b_{i}} \equiv c_{i}, i=0,1,2$ and $c_{0} \neq c_{1} \neq c_{2}$. To show the existence of eigenvalues of the related self-adjoint Toeplitz operator $T_{\phi}$ on $H^{2}\left(d m_{a}\right)$, without loss of generality, one can assume that $c_{2}=-1, c_{1}=1$, and $c_{0}=c$. Let us assume that $1<c$. If $\tau$ is the B-period matrix of the marked double $X$ of $D$, then we have the following. 


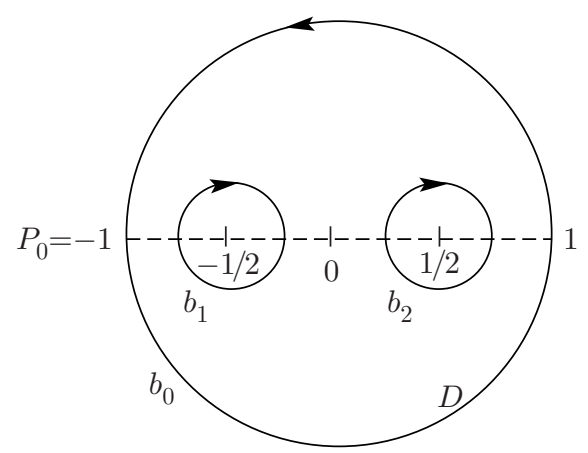

FiguRE 5.

(i) For any $t$ in $(-1,1)$,

$$
\begin{aligned}
\theta\left(t_{a}+\frac{1}{2 \pi} \int_{\partial D} \log (\phi-t) d \vec{w}\right) & =\theta\left(t_{a}+\frac{1}{2 \pi} \int_{\partial D} \log |\phi-t| d \vec{\nu}+\frac{1}{2} \tau\left[\begin{array}{l}
0 \\
1
\end{array}\right]\right) \\
& =F\left(t_{a}+\frac{1}{2 \pi} \int_{\partial D} \log |\phi-t| d \vec{\nu}\right)
\end{aligned}
$$

Since

$$
\begin{aligned}
\underline{x}(t) & =t_{a}+\frac{1}{2 \pi} \int_{\partial D} \log |\phi-t| d \vec{\nu} \\
& =t_{a}+\frac{1}{2 \pi} \int_{b_{0}} \log |c-t| d \vec{\nu}+\frac{1}{2 \pi} \int_{b_{1}} \log |1-t| d \vec{\nu}+\frac{1}{2 \pi} \int_{b_{2}} \log |1+t| d \vec{\nu} \\
& =t_{a}+\frac{1}{2 \pi} \log |c-t| \int_{b_{0}} d \vec{\nu}+\frac{1}{2 \pi} \log |1-t| \int_{b_{1}} d \vec{\nu}+\frac{1}{2 \pi} \log |1+t| \int_{b_{2}} d \vec{\nu},
\end{aligned}
$$

for $t$ near -1 , in the gap $(-1,1)$, the path $\underline{x}(t)$ can be written as

$$
\underline{x}(t)=\underline{r}(t)+\frac{1}{2 \pi} \log |1+t| \int_{b_{2}} d \vec{\nu}=\underline{r}(t)+\frac{1}{2} \log |1+t|\left[\begin{array}{c}
P_{12} \\
P_{22}
\end{array}\right],
$$

where $\underline{r}(t)=\left(r_{1}(t), r_{2}(t)\right)$ and the $r_{i}(t)(i=1,2)$ are bounded. Since $\log |1+t| \rightarrow$ $-\infty$, as $t \rightarrow(-1)^{+}$,

$$
\underline{x}(t)=\left[\begin{array}{l}
x_{1}(t) \\
x_{2}(t)
\end{array}\right] \rightarrow\left[\begin{array}{l} 
\pm \infty \\
-\infty
\end{array}\right], \text { as } t \rightarrow(-1)^{+} .
$$

Similarly,

$$
\underline{x}(t)=\left[\begin{array}{l}
x_{1}(t) \\
x_{2}(t)
\end{array}\right] \rightarrow\left[\begin{array}{l}
-\infty \\
\pm \infty
\end{array}\right], \quad \text { as } t \rightarrow 1^{-} .
$$

Therefore, there exist two infinite sequences of eigenvalues in the gap $(-1,1)$.

(ii) For any $t$ in $(1, c)$,

$$
\begin{aligned}
& \theta\left(t_{a}+\frac{1}{2 \pi} \int_{\partial D} \log (\phi-t) d \vec{w}\right) \\
& \quad=\theta\left(t_{a}+\frac{1}{2 \pi} \int_{\partial D} \log |\phi-t| d \vec{\nu}+\frac{1}{2} \tau\left[\begin{array}{l}
1 \\
1
\end{array}\right]\right)=H\left(t_{a}+\frac{1}{2 \pi} \int_{\partial D} \log |\phi-t| d \vec{\nu}\right) .
\end{aligned}
$$


Since

$$
\begin{aligned}
\underline{x}(t) & =t_{a}+\frac{1}{2 \pi} \int_{\partial D} \log |\phi-t| d \vec{\nu} \\
& =t_{a}+\frac{1}{2 \pi} \int_{b_{0}} \log |c-t| d \vec{\nu}+\frac{1}{2 \pi} \int_{b_{1}} \log |1-t| d \vec{\nu}+\frac{1}{2 \pi} \int_{b_{2}} \log |1+t| d \vec{\nu} \\
& =t_{a}+\frac{1}{2 \pi} \log |c-t| \int_{b_{0}} d \vec{\nu}+\frac{1}{2 \pi} \log |1-t| \int_{b_{1}} d \vec{\nu}+\frac{1}{2 \pi} \log |1+t| \int_{b_{2}} d \vec{\nu},
\end{aligned}
$$

for $t$ near $c$, in the gap $(1, c)$, the path $\underline{x}(t)$ can be written as

$$
\underline{x}(t)=\underline{r}(t)+\frac{1}{2 \pi} \log |c-t| \int_{b_{0}} d \vec{\nu}=\underline{r}(t)-\frac{1}{2} \log |c-t|\left[\begin{array}{c}
P_{11}+P_{12} \\
P_{12}+P_{22}
\end{array}\right],
$$

where $\underline{r}(t)=\left(r_{1}(t), r_{2}(t)\right)$ and the $r_{i}(t)(i=1,2)$ are bounded. Since $\log |c-t| \rightarrow$ $-\infty$, as $t \rightarrow c^{-}$,

$$
\underline{x}(t)=\left[\begin{array}{l}
x_{1}(t) \\
x_{2}(t)
\end{array}\right] \rightarrow\left[\begin{array}{l}
+\infty \\
+\infty
\end{array}\right], \text { as } t \rightarrow c^{-} .
$$

Therefore, there exists an infinite sequence of eigenvalues in the gap $(1, c)$.

\section{REFERENCES}

[1] M. B. Abrahamse, Toeplitz operators in multiply connected regions, Amer. Jour. Math. 96 (1974), 261-297. MR 50:14333

[2] G. Akbari Estahbanati (Cyrus P. Aryana), Riemann surfaces and Toeplitz operators on multiply connected planar regions, Dissertation, University of Georgia, 1993.

[3] K. F. Clancey, Toeplitz operators on multiply connected domains and theta functions, Operator Theory: Adv. and Appl. 35 (1988), 311-355. MR 91f:47038

[4] K. F. Clancey, On the spectral character of Toeplitz operators on multiply connected domains, Trans. Amer. Math. Soc., vol. 323 (1991), 897-910. MR 91f:47039

[5] K. F. Clancey, Representing measures on multiply connected planar domains, Illinois J. Math., vol. 35 (1991), 286-311. MR 92e:46110

[6] H. M. Farkas and I. Kra, Riemann Surfaces, Springer-Verlag, New York, 1992. MR 93a:30047

[7] J. D. Fay, Theta Functions on Riemann Surfaces, Lecture Notes in Mathematics No. 352, Springer-Verlag, New York, 1973. MR 49:569

[8] J. D. Pincus and J. Xia, Symmetric and self-adjoint Toeplitz operators on multiply connected planar domains, J. Funct. Anal. 59 (1984), 397-444. MR 87i:47038

Department of Mathematical Sciences, Saginaw Valley State University, University Center, Michigan 48710

E-mail address: aryana@svsu.edu

Department of Mathematics, University of Louisville, Louisville, Kentucky 40292

E-mail address: k.clancey@louisville.edu 